\title{
Utilization of psychiatric rehabilitation services and influencing factors among people with psychotic disorders in rural communities of Guangxi, China
}

\author{
Hongye Luo ${ }^{1,2}$, Edward B. McNeil ${ }^{2}$, Qiming Feng ${ }^{1}$, Hongheng Li ${ }^{1}$, Qiang Chen ${ }^{3}$, Xianjing Qin ${ }^{1}$, Jun Feng ${ }^{1}$
} and Sawitri Assanangkornchai ${ }^{*}$ (i)

\begin{abstract}
Objectives: To identify the rate and predictors of utilization of rehabilitation services among people with psychotic disorders in rural communities of Guangxi.

Study design: A cross-sectional survey was conducted among individuals with schizophrenia or other psychoses (severe mental disorder, ICD10: F20-F29), aged over 15 years, and their care-givers in Guangxi, China. Trained village doctors located individuals known to them and suspected as having schizophrenia or other psychoses within the target areas and recruited them into the study. Data on demographic characteristics, clinical symptoms and functions, treatment history, and reasons, if any, for non-utilization of mental health service were collected. Logistic regression was used to determine associated factors for utilization of mental health services.

Results: A total of 424 individuals experiencing psychosis (mean age $41.4 \pm 13.0$ years, $60.6 \%$ male) and 319 caregivers (mean age $55.3 \pm 14.2$ years) were interviewed. The median duration of disease was 13.4 years. $83.0 \%$ of patients had never used rehabilitation services. Greater use of rehabilitation was associated with having a non-organic disorder $(\mathrm{OR}=11.6,95 \% \mathrm{Cl}=1.6-86.0)$ and living with a caregivers $(\mathrm{OR}=3.2,95 \% \mathrm{Cl}=1.2-8.3)$. The top three reasons for not using rehabilitation services were lack of awareness (57.1\%), lack of money (14.2\%) and lack of belief in the service (12.8\%).

Conclusions: These findings indicate a high unmet need for psychiatric rehabilitation services among people with psychotic disorders in rural areas of Guangxi. Strategies such as outreach programme and collaborative and partnership network with the local community are needed to encourage people with psychotic disorders in rural communities to increase their utilization of rehabilitation services.
\end{abstract}

Keywords: Severe mental disorder, People with psychotic disorders, Psychiatric rehabilitation services, Influencing factors, Rural communities

\section{Background}

Mental disorders occur at high rates in every country, and have already become a global challenge, especially in fast-growing developing countries such as China [1,

\footnotetext{
*Correspondence: savitree.a@psu.ac.th

${ }^{2}$ Epidemiology Unit, Faculty of Medicine, Prince of Songkla University, Hat Yai, Songkhla, Thailand

Full list of author information is available at the end of the article
}

2]. The second national schizophrenic epidemiological investigation of China in 1993 showed that more than 83 million were living with mental disorders, 16 million suffered from severe mental disorders such as schizophrenia, and two-thirds lived in rural areas [3].

Psychiatric rehabilitation is an important part of a mental health service. It promotes recovery, community integration, and improves quality of life for patients who 
have been diagnosed with any mental health condition that seriously impairs their ability to lead meaningful lives. If the patients received rehabilitation their social function will be improved which in turn will decrease the burden of caregivers [4]. Psychiatric rehabilitation services focus on helping patients develop skills and access resources needed to increase their ability to be successful and satisfied in the living, working, learning, and social environments of their choice [5]. They are especially related to the improvement of functioning and quality of life [6]. Additionally, psychiatric rehabilitation aims to reintegrate patients with severe mental disorders into society and help them to live fulfilling lives $[7,8]$. Psychiatric rehabilitation services include medications for treatment-resistant psychosis and affective disorders, physical health promotion, psychological interventions such as cognitive behavioral therapy and family interventions, occupational therapy, and supported employment [9].

The practice of psychiatric rehabilitation over the last few decades has undergone gradual refinement and sophistication, which has resulted in a multitude of intervention programmes in the United States, Canada and the United Kingdom [9-12]. However, to a large extent, it has been restricted to developed countries. Its importance has not been recognized in many developing countries, and its practice is still rare compared to the use of medicine to treat patients. Although mental health is part of the primary healthcare system, very little active psychiatric treatment or psychiatric rehabilitation happens especial in most low- and middle-income countries [6]. Evidently, large differences between countries may exist with respect to the availability and organization of rehabilitation services although most jurisdictions would argue that not all people living with severe mental disorders such as schizophrenia or delusional disorders require rehabilitation at any one time [13]. There is little in terms of documentation of their activities and limited research on the utilization of psychiatric rehabilitation services in low- and middle-income countries [6]. According to previous studies, $40 \%$ of patients with schizophrenia did not have any vocational or educational perspectives at discharge from hospital in Belgium $[14,15]$. Studies conducted in Israel showed only about $15-20 \%$ of the estimated number of the eligible population received the service $[16,17]$. The report of Nepal showed 1-20\% of people with psychotic disorders in outpatient facilities received psychiatric rehabilitation service $[18,19]$.

Lack of utilization of mental health services can lead to irreversible conditions. However, mental health services in China are mostly provided by psychiatric specialists in large hospitals. In addition, most psychiatrists and psychiatric specialist hospitals are located in cities. Guangxi is located in southern China. Very few mental health services exist in this relatively underdeveloped province, especially in rural areas. The prevalence of schizophrenia in rural Guangxi was 9.2\% [20,21]. Utilization of mental health services in rural communities of Guangxi may be inadequate.

WHO stated there remains a group of patients with psychotic disorders who do not recover adequately to be discharged home following efficient psychiatric services [18]. Lack of access to high-quality services also exists in the wealthiest countries. In the USA, less than $5 \%$ of people with severe psychiatric disorders can access high-quality psychiatric rehabilitation [10]. It has been estimated that around 1\% of people in England with schizophrenia received inpatient rehabilitation at any one time [11]. It showed that rehabilitation services are far from generally available. A study in China reported that the utilization rate of psychiatric rehabilitation services by patients with schizophrenia was only $1.4 \%$, and most of the patients never continued with the service once they went back into the community [22]. Another study in Shanghai reported that $9.2 \%$ of patients with severe mental disorders received psychiatric rehabilitation [23].

Very few surveys have been conducted attempting to understand the situation of psychiatric rehabilitation service utilization [14, 20-27], thus, little information is available on the predictors of these services, particularly community-based ones among patients with severe mental disorders. One study reported that family's expectations and patient's negative symptoms significantly correlated with rehabilitation needs and utilization [28]. Two other studies found that age, family structure, shame of disease and self-consciousness were associated with psychiatric rehabilitation utilization, and lack of district rehabilitation facility resources and personnel influenced the provision of psychiatric rehabilitation services [29, 30].

Over half of the people with schizophrenia are not receiving proper care [2]. Absence of a national mental health policy or programme in many LAMIC makes it unrealistic to expect that psychosocial interventions would be high on their agenda. It is therefore crucial for the government to formulate precise mental health policies to guide the practices to address these pressing issues [31]. Based on this point, the national mental health work plan of China (2015-2020) explicitly states that efforts are needed to improve psychiatric rehabilitation services. Every province must establish and develop communitybased rehabilitation programs for mental disability patients. The plan demands at least one mental health service center be built in every county. As an important part of the mental health service system, county mental health center functions should cover the provision of psychiatric rehabilitation services [32]. Concerns about the 
levels of rehabilitation service received by those with serious mental illness have been growing as a result of recent changes in social welfare policy and mental health care delivery systems [22]. Assessing the utilization forms an important constituent in the planning of Mental Health Services [23]. Identifying the current psychiatric rehabilitation utilization rate and related barriers is a critical first step in planning, developing and targeting interventions to address the emerging needs of people with psychotic disorders and improve the appropriateness of psychiatric rehabilitation service and health outcomes of those with serious mental illness.

According to the current status of psychiatric rehabilitation research $[14,15,33,34]$, two questions are addressed: (1) what are the rates of psychiatric rehabilitation service utilization among persons with severe mental disorders? And (2) what factors are associated with the utilization of psychiatric rehabilitation services among these patients? In this study, we sought to improve our understanding of the utilization of psychiatric rehabilitation services among persons with severe mental disorders. Results of this study can be used to assist policy makers formulate more appropriate mental health strategies in Guangxi Zhuang Autonomous Region. Patient's symptoms have previously been shown to be significantly correlated with rehabilitation needs $[2,30,31]$. Thus, the relationship between the uses of psychiatric rehabilitation services with patient symptoms was also examined.

\section{Methods}

\section{Sample selection}

A cross-sectional survey was conducted to investigate the utilization of psychiatric rehabilitation among individuals aged over 15 years with severe mental disorders and their caregivers during June to September, 2016. The survey employed a multistage stratified, clustered sampling scheme to select 118 villages from nine towns in three counties of Guangxi Autonomous Region, China. Altogether, 12 psychiatrists, 36 township hospital nurses or doctors, 118 village doctors and 20 research assistants were trained and participated in the survey. Liujiang county, Liucheng county and Xiangzhou county were selected as sample counties. Three townships were selected from each county based on the distance and population size. Four most populous administrative villages were chosen from each township (If the village population is less than 1000, the neighbor village was chosen to increase the population). All the natural villages of the administrative village were included in the sample. Finally 118 villages from three counties were included in this study, including 36 villages from Lijiang county, 41 villages from Liucheng county and 40 villages from Xiangzhou county with 47,929 residents altogether. All individuals suspected as having schizophrenia or other psychoses within the target area reported by the village doctors were screened by the township nurses and diagnosed by the psychiatrists. A total of 424 severe mental disorder patients were diagnosed and interviewed, including 185 patients from Liujiang county, 115 patients from Liucheng county and 124 patients from Xiangzhou county.

An indigenous field worker sampling method was employed in which village doctors were trained to recruit participants. These village doctors located individuals known to them and suspected as having schizophrenia or other psychoses within the target area and recruit them into the study. Multiple sites and recruitment networks were chosen to ensure a wide coverage of the target population and to reduce volunteer bias.

\section{Study participants}

Individuals aged 15 years or over, residing in the study area for at least 6 months prior to the study period were eligible. In this study, severe mental disorders included schizophrenia, schizotypal, and delusional disorders (International Statistical Classification of Diseases and Related Health Problems (ICD-10) codes F20-F29) and other psychoses (e.g. organic psychoses) which are consistent with the "Severe mental illness treatment and management specification" launched by the Chinese Government. All patients were assessed for a definitive diagnosis by qualified psychiatrists.

\section{Interview and measures}

After providing written informed consent, each participant met in person with a research staff for an interview that lasted approximately half an hour. Data on demographic characteristics, clinical symptoms, treatment history, use of psychiatric rehabilitation services and, if appropriate, reasons for not utilizing the service were elicited. Psychiatrists conducted clinical interviews to evaluate the severity of a patient's condition using the positive and negative syndrome scale (PANSS) [35]. Scores range from 7 to 49 for positive and negative scales and from 16 to 112 for the general scale and higher scores represent more severe symptoms. The psychiatrists also inquired about the caregiver's information such as age, gender, education level and marital status. Each participant was given $\$ 10$ as compensation for their time.

Utilization of psychiatric rehabilitation services was defined as any use of psychiatric rehabilitation programmes by the patients within 12 months prior to the survey. This included social skills training, cognitive/ cognitive-behavioral therapy, family psycho education and vocational rehabilitation. In this study, the utilization of psychiatric rehabilitation services included both inpatient and community-based services. 
Ethics

The study was approved by the Ethics Committee for Research in Human Subjects, Faculty of Medicine, Prince of Songkla University. All participants provided written informed consent to participate in the study. Data collection was conducted in private and participants were assured that any information disclosed would be treated in strict confidence. All data on patients were saved and analyzed anonymously. The China Medical Board approved the study.

\section{Data analysis}

Descriptive statistics were used to summarize the demographic data of the participants and their caregivers. Pearson's Chi square test was used to compare differences in socio-demographic variables, cause of illness and disease duration between those utilizing and not utilizing psychiatric rehabilitation services while Student's independent t-test was used to compare differences in positive, negative and general symptom scores. Based on some previous studies, patients' characteristics, including demographics, course of illness, type of symptoms (positive or negative) and type of psychotic disorders as well as caregivers' characteristics were associated with the use of treatment and rehabilitation services [21], we therefore looked at the associations between each of these variables in the univariate analyses. The variables with $p$ value $\leq 0.20$ in the univariate analyses were included in the initial model. To identify predictive factors influencing the utilization of psychiatric rehabilitation services, multivariate logistic regression models were then used to calculate adjusted odd ratios (OR) and $95 \%$ confidence intervals $(\mathrm{CI})$. The significance level was set at $\mathrm{p}<0.05$. All data were analyzed using $\mathrm{R}$ version 3.3.2.

\section{Results}

A total of 424 severe mental disorder patients (60.6\% male) aged over 15 years and 319 caregivers were interviewed. The mean age of the patients was $41.4 \pm 13.0$ years and the caregiver was $55.3 \pm 14.2$ years. Most (72.4\%) patients were Zhuang ethnicity, completed elementary $(38.4 \%)$ or junior high school $(37.7 \%)$ and $49.5 \%$ were single. $63.4 \%$ were engaged in agricultural work while $30.0 \%$ were not currently employed and $44.8 \%$ had a low per-capita annual family income level $(<981$ USD). The median duration of illness was 13.4 (Interquartile range (IQR): 6.45-67) years. Median scores of positive, negative and general symptoms of the PANSS were 12,15 and 25 , respectively. The utilization rate for any rehabilitation service was $17.0 \%$. Demographic variables are summarized in Table 1.
Table 1 Socio-demographic and clinical characteristics of the participants and their caregivers

\begin{tabular}{|c|c|c|}
\hline Characteristic & Frequency & Percent \\
\hline \multicolumn{3}{|l|}{ Gender } \\
\hline Male & 257 & 60.6 \\
\hline Female & 167 & 39.4 \\
\hline \multicolumn{3}{|l|}{ Ethnicity } \\
\hline Zhuang & 307 & 72.4 \\
\hline Han and other & 117 & 27.6 \\
\hline \multicolumn{3}{|l|}{ Age group (years) } \\
\hline$<30$ & 91 & 21.5 \\
\hline $30-39$ & 124 & 29.3 \\
\hline $40-49$ & 113 & 26.7 \\
\hline$\geq 50$ & 96 & 22.6 \\
\hline \multicolumn{3}{|l|}{ Education level } \\
\hline Illiterate & 67 & 15.8 \\
\hline Elementary school & 163 & 38.4 \\
\hline Junior high school & 160 & 37.7 \\
\hline High school or higher & 34 & 8.0 \\
\hline \multicolumn{3}{|l|}{ Marital status } \\
\hline Single & 210 & 49.5 \\
\hline Married & 187 & 44.1 \\
\hline Divorced or widowed & 27 & 6.4 \\
\hline \multicolumn{3}{|l|}{ Occupation } \\
\hline Unemployed & 127 & 30.0 \\
\hline Farmer & 269 & 63.4 \\
\hline Other & 28 & 6.6 \\
\hline \multicolumn{3}{|l|}{ Annual family income level (US dollars) } \\
\hline High (> 1802) & 74 & 17.5 \\
\hline Middle (981-1802) & 160 & 37.7 \\
\hline $\operatorname{Low}(<981)$ & 190 & 44.8 \\
\hline \multicolumn{3}{|l|}{ Cause of severe mental disease } \\
\hline Organic psychoses & 50 & 11.8 \\
\hline Other non-organic severe mental disorders & 374 & 88.2 \\
\hline \multicolumn{3}{|l|}{ Disease duration (years) } \\
\hline$<10$ & 158 & 37.3 \\
\hline $10-19$ & 131 & 30.9 \\
\hline$\geq 20$ & 135 & 31.8 \\
\hline \multicolumn{3}{|l|}{ PANSS score, median (IQR) } \\
\hline Positive scale & $12(7-14)$ & \\
\hline Negative scale & $15(7-48)$ & \\
\hline General psychopathology & $25(16-76)$ & \\
\hline \multicolumn{3}{|l|}{ Caregiver } \\
\hline Yes & 352 & 83.0 \\
\hline No & 72 & 17.0 \\
\hline \multicolumn{3}{|l|}{ Caregiver's gender } \\
\hline Male & 195 & 61.1 \\
\hline Female & 124 & 38.9 \\
\hline
\end{tabular}

PANSS positive and negative syndrome scale, SD standard deviation 
There were no significant differences between those who ever utilized and those who had never utilized any rehabilitation service in terms of gender, ethnicity, age group, marital status, occupation, annual family income, disease duration and caregiver's gender. However, statistically significant differences were found in educational level, type of psychoses, having a caregiver and PANSS scores in the univariate analyses as shown in Table 2. Patients with schizophrenia and other nonorganic mental disorders utilized psychiatric rehabilitation services more than those with organic severe mental disorders $(\mathrm{p}=0.005)$. Patients who had a caregiver used the service more than those without a caregiver $(\mathrm{p}=0.037)$. In addition, those who did not utilize psychiatric rehabilitation services had significantly higher positive symptoms $(p=0.024)$, negative symptoms $(p=0.016)$ and general symptoms $(p=0.003)$ from the PANSS.

From the results of the multivariate logistic regression model shown in Table 3, two factors were associated with utilization of rehabilitation: having a non-organic mental disorder $(\mathrm{OR}=11.6,95 \% \mathrm{CI}=1.6,86.0)$ and having a caregiver $(\mathrm{OR}=3.2,95 \% \mathrm{CI}=1.2,8.3)$.

As shown in Table 4, the top three reasons for not utilizing rehabilitation services were lack of awareness (57.1\%), excessive cost (14.2\%) and lack of belief in the service (12.8\%).

\section{Discussion}

This study describes the utilization of psychiatric rehabilitation services and associated factors among people with severe mental disorder in Guangxi rural communities. The utilization rate was very low, only $17.0 \%$, which is consistent with previous studies in China and in other developing countries (1-40\%) [6, 19, 22, 23, 25-27, 36]. Psychiatric rehabilitation utilization rates are also low in developed countries [37]. Reasons for the low utilization of psychiatric rehabilitation services are multifactorial. Some of the factors in developing countries are largely similar to developed countries, such as the economic problems, defective national insurance system, high social stigma of mental illness, lack staff trained in basic principles of psychosocial rehabilitation, the aim of rehabilitation is not fully understood, lack of national mental health policy $[38,39]$. Mental health care in China is still predominantly institution based and focuses mainly on medical treatment. Psychiatric rehabilitation services have not received enough attention from the government. There is a scarcity of policies that have led to effective strategies and intervention programs to support individuals seeking treatment. Guangxi is an undeveloped province of China, with a lack of mental health resources, especially personnel and training, which has
Table 2 Comparison of factors between patients who utilized rehabilitation services and those who did not

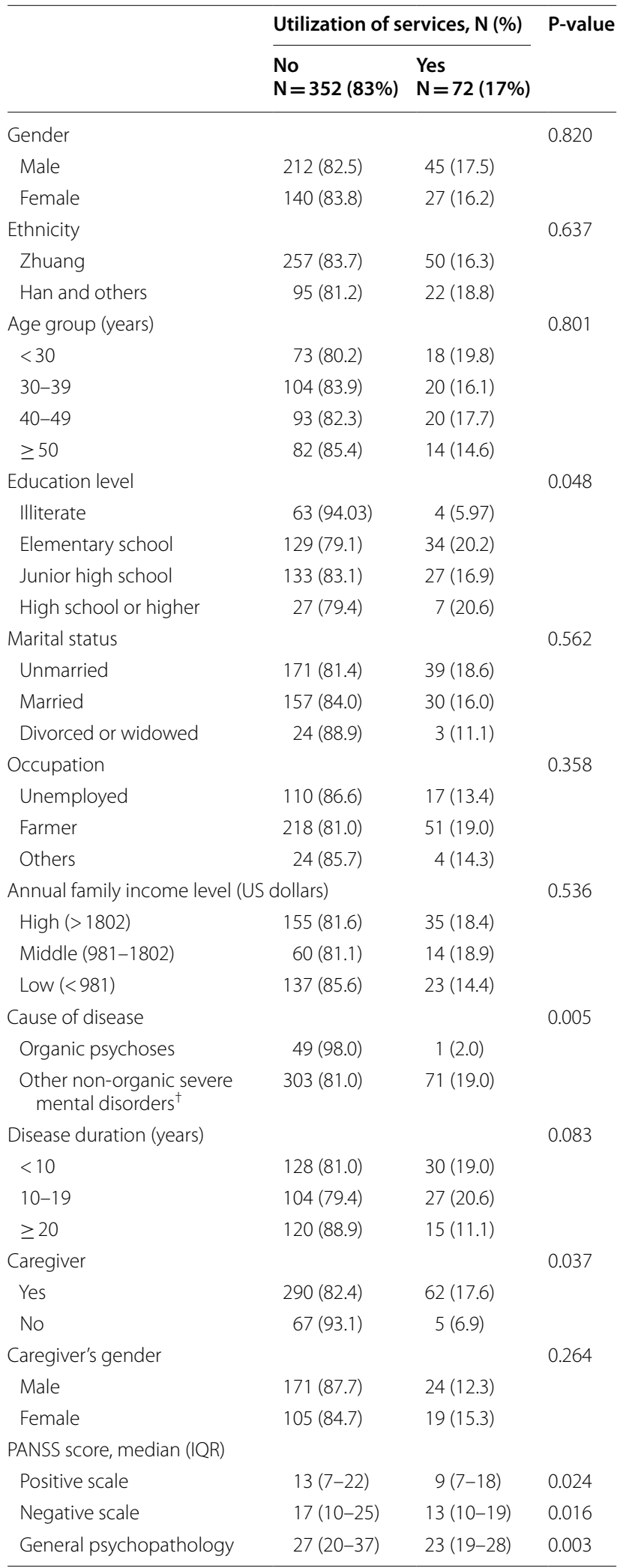

PANSS positive and negative syndrome scale, SD standard deviation, IQR interquartile range

+ Schizophrenia and other non-organic mental disorders 
Table 3 Logistic regression model presenting predictors of psychiatric rehabilitation service utilization among severe mental disorder patients

\begin{tabular}{|c|c|c|}
\hline & Crude OR (95\% Cl) & Adjusted OR (95\% Cl) \\
\hline Female gender (vs male) & $0.9(0.5-1.5)$ & \\
\hline $\begin{array}{l}\text { Zhuang ethnicity (vs Han } \\
\text { and others) }\end{array}$ & $0.8(0.5-1.5)$ & \\
\hline \multicolumn{3}{|l|}{ Age group (years): ref. $=<30$} \\
\hline $30-39$ & $0.8(0.4-1.6)$ & \\
\hline $40-49$ & $0.9(0.4-1.8)$ & \\
\hline$\geq 50$ & $0.7(0.3-1.5)$ & \\
\hline \multicolumn{3}{|c|}{ Education level: ref. $=$ Illiterate } \\
\hline Elementary school & $4.2(1.4-12.2)$ & \\
\hline Junior high school & $3.2(1.1-9.5)$ & \\
\hline High school or higher & $4.1(1.1-15.1)$ & \\
\hline \multicolumn{3}{|c|}{ Marital status: ref.=unmarried } \\
\hline Married & $0.8(0.5-1.4)$ & \\
\hline Divorced or widowed & $0.6(0.2-1.9)$ & \\
\hline \multicolumn{3}{|c|}{ Occupation: ref. = unemployed } \\
\hline Farmer & $1.5(0.8-2.7)$ & \\
\hline Other & $1.1(0.3-3.5)$ & \\
\hline \multicolumn{3}{|c|}{ Annual family income level: ref. $=$ low } \\
\hline Middle & $0.7(0.4-1.3)$ & \\
\hline High & $1.0(0.5-2.1)$ & \\
\hline \multicolumn{3}{|l|}{ Cause of disease } \\
\hline $\begin{array}{l}\text { Other non-organic } \\
\text { severe mental } \\
\text { disorders (vs organic } \\
\text { psychoses) }\end{array}$ & $11.5(1.6-84.6)$ & $11.6(1.6-86.0)$ \\
\hline Disease duration (years) & $1.0(0.9-1.0)$ & \\
\hline \multicolumn{3}{|l|}{ PANSS score } \\
\hline Positive scale & $1.0(0.9-1.0)$ & \\
\hline Negative scale & $1.0(0.9-1.0)$ & \\
\hline $\begin{array}{l}\text { General psychopathol- } \\
\text { ogy }\end{array}$ & $1.0(0.9-1.0)$ & \\
\hline \multicolumn{3}{|l|}{ Caregiver } \\
\hline Yes (vs no) & $6.2(2.0-18.3)$ & $3.21(1.2-8.3)$ \\
\hline \multicolumn{3}{|l|}{ Caregiver's gender } \\
\hline Female (vs male) & $1.3(0.6-2.4)$ & \\
\hline
\end{tabular}

PANSS positive and negative syndrome scale, $\mathrm{OR}$ odds ratio, $\mathrm{Cl}$ confidence interval, ref reference group for comparison

+ Schizophrenia and other non-organic mental disorders

led to a lack of service provision. In addition, the psychiatric rehabilitation service is not covered by the current medical insurance system, making treatment unaffordable for many patients.

Compared with previous studies in China [22, 23, 2527], the rate of patients who used psychiatric rehabilitation services was higher in this study. Li reported a rate as low as $1.0 \%$ from a national survey [26] while Zhang Jianling reported a rate of $9.2 \%$ in a district of Shanghai [22]. One possible explanation for our higher utilization
Table 4 Reasons for non-utilization of psychiatric rehabilitation services among severe mental disorder patients, $\mathbf{N}$ $=352$

\begin{tabular}{lcc}
\hline Reason & Frequency & Percent \\
\hline Lack of awareness of the service & 201 & 57.1 \\
Service is too expensive & 50 & 14.2 \\
Lack of belief in the service & 45 & 12.8 \\
Refused to seek or utilize the service & 36 & 10.2 \\
Don't know the location of the service & 20 & 5.7 \\
\hline
\end{tabular}

rate may be because psychiatric rehabilitation services in this study included services received in hospital, while the other studies included only community psychiatric rehabilitation services. Almost all of the patients received psychiatric rehabilitation services during their hospitalization period. This means when patients return to the community, they rarely used the psychiatric rehabilitation service available to them. Most patients with severe mental disorders now live in the community and should receive treatment from community-based services. Strengthening community based rehabilitation is thus urgently needed [30].

This study found that patients with organic psychoses were less likely to use psychiatric rehabilitation services. It is likely that this group of patients is lack of the ability to express their needs adequately or their caregivers did not believe the psychiatric rehabilitation service could work on the patients, thus did not seek psychiatric rehabilitation services [40]. Several studies found that patients and their family members were unable to express their psychiatric rehabilitation needs $[6,32,41]$. In addition, the positive capacity of people with severe mental illnesses are typically ignored by society including family members, especially for those with organic psychoses, and their families do not believe the patients can return to normal lives. Family members usually take a skeptical attitude for the treatment and rehabilitation, which often leads to the patients giving up rehabilitation service [26, 41]. Family's ignorance towards the illness may contribute to the low utilization rate of psychiatric rehabilitation services of these patients.

Patients with caregivers were more likely to utilize rehabilitation services compared to those without. Caregivers constitute the major support system bearing the responsibility for patient care [39] and influence patients to receive services that they need. Our finding raises the important role of caregivers, who could help patients towards their needs. The local government may attempt to assign caregivers, such as a village doctor, neighbor or volunteer to help patients who live alone. It also indicates 
that interventions such as caregivers training should be implemented to promote caregiver supervision of the patients so that they comply with medication and receive the required rehabilitation services.

This study found that participants who did not utilize psychiatric rehabilitation services had higher scores for negative symptoms, higher scores for positive symptoms and higher scores for general psychopathology compared to those who did not utilize these services. Psychiatric rehabilitation service users often have prominent 'negative' symptoms that may impair their motivation and organizational skills to manage daily activities. This places them at risk of self-neglect. Many also have on-going 'positive' symptoms which have not responded fully to medication and can make communication and engagement difficult. They are lack of the ability to express their needs thus their caregiver and family members are required to help them to access the psychiatric rehabilitation service. However, for some reasons such as the low education level or lack of the awareness, patients and their family can't express their needs adequately. However, those differences were not shown in the multivariate analysis, indicating that the severity of the illness was not associated with utilization after adjusting for cause of disease and presence of a caregiver. This finding was consistent with the study in China reported by $\mathrm{Li}$ [26]. Several studies have shown that meeting the needs of psychiatric patients might be an effective strategy to control their symptoms [28, 42]. But other studies have shown that the severity of symptoms correlates well with patients who express a need for rehabilitation [43]. Thus, whether patients with high scores for negative, positive and general symptoms express a need to have psychiatric rehabilitation requires further exploration in future studies.

The top three reasons for not utilizing psychiatric rehabilitation services were lack of awareness (57\%), lack of money (14\%) and lack of belief in the service (13\%). It seems that the patients and their family members may not be aware of the importance of the psychiatric rehabilitation services available to them, and this represents a significant barrier for patients and their families. This lack of awareness and belief in the importance of psychiatric rehabilitation services more likely led to the low utilization rate found in this study. More efforts are needed to make mentally ill patients more aware of the importance of psychiatric rehabilitation services for their long-term recovery. Regarding the cost of treatment, psychiatric rehabilitation services are not covered by the current medical insurance system [44], which may partly explain why many patients with severe mental disorders who are covered by medical insurance still could not afford to receive treatment. Efforts are warranted to strengthen legislation that improves insurance coverage of psychiatric rehabilitation services.

Results of this study should be interpreted with the following three main limitations in mind. First, since the sample was selected from only three counties, the results may be not representative of the general situation in Guangxi. Second, an indigenous field worker sampling method was employed, thus we may have missed some less severe mental disorder patients. Since the study was a community-based survey, homeless or some institutionalized patients may have been inadvertently missed. Hence, caution should be made when generalizing these findings in formulating suggestions for mental health services or policies in Guangxi. Finally, we did not evaluate the perspective of the service providers, such as their resources and skills. Further studies exploring the effect of service providers on psychiatric rehabilitation utilization are recommended.

\section{Conclusion}

The findings of this study highlight a low utilization of psychiatric rehabilitation services in Guangxi. It is important that the government formulate relevant mental health policies and incorporate them into public health and social policy that suits the needs of severe mental disorder patients and their families. Psychiatric rehabilitation service operates as a whole system which includes many other institutions and organizations. Therefore, the collaborative and partnership network should be developed and the links with local community resource to facilitate services should be strengthened. Psychiatric rehabilitation service worker training programs and psychiatric rehabilitation intervention programs as done in other developed countries such as US, Canada, UK and Australia [40] should be developed.

\section{Authors' contributions}

Conception or design of the work: HL, SA, QF. Data collection: HL,QC, XQ, JF. Data analysis and interpretation: HL, EBMCN, SA. Drafting the article: HL. Critical revision of the article: SA, EBMcN. All authors read and approved the final manuscript

\section{Author details \\ ${ }^{1}$ Information and Management School, Guangxi Medical University, Nan- ning, Guangxi Zhuang Autonomous Region, China. ${ }^{2}$ Epidemiology Unit, Faculty of Medicine, Prince of Songkla University, Hat Yai, Songkhla, Thailand. ${ }^{3}$ Guangxi Brain Hospital, Liuzhou, Guangxi Zhuang Autonomous Region, China.}

\section{Acknowledgements}

The authors wish to thank the Guangxi Mental health center and Guangxi Brain Hospital who generously offered their general support and assistance during the program. As would also like to thank the township hospital staff and the village doctors for their help in data collection, and would like to acknowledge the support from the China Medical Board (CMB) and Natural Science Foundation of China (NSFC).

Competing interests

The authors declare that they have no competing interests. 


\section{Availability of data and materials}

The datasets generated and/or analysed during the current study are not publicly available as participants are severe mental disorder patients and they may be easily identified in their respective communities, but are available from the corresponding author on reasonable request.

\section{Consent for publication}

Not applicable.

\section{Ethics approval and consent to participate}

Ethical approval for the study was obtained from the Prince of Songkla University Research Ethics Committee in April 2016 (Approval Number: 59029185) and the additional site-specific approval for the regional city was obtained in August 2016. All participants provided written informed consent to participate.

\section{Funding}

The study was supported by China Medical Board (CMB)-OC Project (Grant Number 14-203) and Natural Science Foundation of China (NSFC) (Item No.: 81460521).

\section{Publisher's Note}

Springer Nature remains neutral with regard to jurisdictional claims in published maps and institutional affiliations.

Received: 15 October 2017 Accepted: 5 April 2018

Published online: 17 April 2018

\section{References}

1. WHO. Integrating mental health into primary care: a global perspective. Geneva: World Health Organization; 2008.

2. WHO. Mental health. Geneva: World Health Organization; 2012.

3. Changhui $C$, et al. Epidemiology investigation for Schizophrenia in seven areas of China. Chin J Psychiatry. 1998;31(2):72-4.

4. Poon A, Joubert L, Mackinnon A, Harvey C. A longitudinal populationbased study of carers of people with psychosis. Epidemiol Psychiatr Sci. 2017;26:265-75.

5. About PRA. Psychiatric rehabilitation association. Archived from the original on 15 February 2015. Accesses 15 Feb 2015.

6. Our History. Psychiatric rehabilitation association. Archived from the original on 7 November 2016. Accessed 17 Nov 2016.

7. Rangaswamy T, Sujit J. Psychosocial rehabilitation in developing countries. Int Rev Psychiatry. 2012;24(5):499-503.

8. Craig T, Garety P, Power P, et al. The Lambeth Early Onset (LEO) Team: randomised controlled trial of the effectiveness of specialised care for early psychosis. BMJ. 2004;329:1067-71.

9. Morgan C, Lappin J, Heslin M, Donoghue K, Lomas B, Reininghaus U, et al. Reappraising the long-term course and outcome of psychotic disorders: the AESOP-10 study. Psychol Med. 2014;44:2713-6.

10. Roessler W. Psychiatric rehabilitation today: an overview. World Psychiatry. 2006;5(3):151

11. Killaspy H. Contemporary mental health rehabilitation. East Asian Archives of Psychiatry. 2014:24(3):89.

12. Psychosocial Rehabilitation Association of Canada. PSR/RPS Canada.

13. Kazdin AE. Addressing the treatment gap: a key challenge for extending evidence-based psychosocial interventions. Behav Res Ther. 2017:88:7-18

14. De Hert M, Detraux J, Peuskens J. Practice of and services for psychosocial rehabilitation of people with schizophrenia in Belgium. Giorn Ital Psicopat. 2010;16:255-65.

15. Klinkenberg WD, Calsyn RJ. Predictors of receipt of aftercare and recidivism among persons with severe mental illness: a review. Psychiatr Serv. 1996;47(5):487-96.

16. Aviram U. Promises and pitfalls on the road to a mental health reform in Israel. Isr J Psychiatry Relat Sci. 2010;47(3):171.

17. Struch N, et al. People with severe mental disorders in Israel: an integrated view of the service systems. Jerusalem: Myers-JDC-Brookdale Institute; 2009.
18. World Health Organization. WHO-AIMS report on mental health system in Nepal. Geneva: World Health Organization; 2007.

19. Kamenov K, et al. Needs and unmet needs for rehabilitation services: a scoping review. Disab Rehab. 2018: 1-11.

20. Bo W, et al. Epidemiology investigation for mental disorder on urban and rural residents in Guangxi Zhuang Autonomous Region. Guangxi Med Univ J. 2010;12(6):951-6.

21. Bo W, et al. Epidemiology investigation of mental disorder in rural area of Guangxi Zhuang Autonomous Region. Mod Prevent Med. 2011;10:1801-5.

22. Zhang J, et al. A study of mental rehabilitation states and its relative factors of patients with schizophrenia in community. Med J Chin People's Health. 2006;18(3):168-71.

23. Zhang Q, et al. The current situation and suggestions of community rehabilitation service resource in Shanghai Changning district. Shanghai J Prevent Med. 2016:28(7):19-24.

24. WHO. Mental health atlas ddsf. Geneva: World Health Organization; 2005.

25. Hogarty GE, et al. Family psychoeducation, social skills training, and maintenance chemotherapy in the aftercare treatment of schizophrenia: I. One-year effects of a controlled study on relapse and expressed emotion. Arch Gen Psychiatry. 1986;43(7):633-42.

26. Li N, et al. Mental health service use among Chinese adults with mental disabilities: a national survey. Psychiatric Serv. 2013;64(7):638-44.

27. Man DW, Law KM, Chung RC. Cognitive training for Hong Kong Chinese with schizophrenia in vocational rehabilitation. Hong Kong Med J. 2012;18(6):18-22.

28. Gandotra S, et al. A preliminary study of rehabilitation needs of in-patients and out-patients with schizophrenia. Indian J Psychiatry. 2004;46(3):244

29. Li D, et al. Measuring perceived rehabilitation needs of people with schizophrenia in mainland China. Admin Policy Ment Health Ment Health Serv Res. 2014;41(5):636-46.

30. Barton R. Psychosocial rehabilitation services in community support systems: a review of outcomes and policy recommendations. Psychiatric Serv. 1999:50(4):525-34

31. Jenkins R. Making psychiatric epidemiology useful: the contribution of epidemiology to government policy. Acta Psychiatr Scand. 2001;103:2-14

32. Li D, Tsui MC, Yuan GZ. China's new Mental Health Law needs to tackle manpower imbalance of professionals in schizophrenia psychosocial rehabilitation. Int J Soc Psychiatry. 2014;60(4):410-1.

33. Wig N Srinivasmurthy R. An approach to organizing rural psychiatric services. A report from WHO project "Strategies for extending mental health care". Geneva: World Health Organization; 1981.

34. Wang PS, Demler O, Kessler RC. Adequacy of treatment for serious mental illness in the United States. Am J Public Health. 2002;92(1):92-8.

35. Kay SR, Fiszbein A, Opfer LA. The positive and negative syndrome scale (PANSS) for schizophrenia. Schizophr Bull. 1987;13(2):261.

36. Phutane $\mathrm{VH}$, et al. Barriers to discharge of patients from a governmentrun day-care psychiatric rehabilitation center in India. Asian J Psychiatry. 2012;5(1):34-7.

37. Cancro R. The underutilization of psychiatric rehabilitation. World Psychiatry. 2006:5(3):159.

38. van Busschbach J, Wiersma D. Does rehabilitation meet the needs of care and improve the quality of life of patients with schizophrenia or other chronic mental disorders? Commun Ment Health J. 2002;38(1):61-70.

39. Hou SY, et al. Exploring the burden of the primary family caregivers of schizophrenia patients in Taiwan. Psychiatry Clin Neurosci. 2008;62(5):508-14.

40. Panel JC. Rehabilitation services for people with complex mental health needs. 2016. https://www.jcpmh.info/.

41. Barbato A. Psychosocial rehabilitation and severe mental disorders: a public health approach. World Psychiatry. 2006;5(3):162.

42. Bell MD, Lysaker PH. Psychiatric symptoms and work performance among persons with severe mental illness. Psychiatric Serv. 1995;46(5):508-10.

43. Shihabuddeen $T$, et al. Rehabilitation needs and disability in persons with Schizophrenia and the needs of the care givers. Delhi Psychiatry J. 2012;15(1):118-21.

44. Xiao S. Critical challenge to Chinese mental health service system. Chin Ment Health J. 2009:23:844-7 (in Chinese) 